\title{
Population pharmacokinetic data analysis of three phase I studies of matuzumab, a humanised anti-EGFR monoclonal antibody in clinical cancer development
}

\author{
K Kuester ${ }^{1,3}$, A Kovar², C Lüpfert ${ }^{2}$, B Brockhaus² and C Kloft", 1,3 \\ 'Department of Clinical Pharmacy, Institute of Pharmacy, Freie Universitaet Berlin, Berlin, Germany; ${ }^{2}$ Department of Human Pharmacology, Merck KGaA, \\ Darmstadt, Germany; ${ }^{3}$ Department of Clinical Pharmacy, Institute of Pharmacy, Martin-Luther-Universitaet Halle-Wittenberg, Halle, Germany
}

\begin{abstract}
A population pharmacokinetic model based on data from three phase I studies was to be developed including a covariate analysis to describe the concentration-time profiles of matuzumab, a novel humanised monoclonal antibody. Matuzumab was administered as multiple I h i.v. infusions with I I different dosing regimens ranging from 400 to $2000 \mathrm{mg}$, q I w-q3w. For analysis, 90 patients with 1256 serum concentration-time data were simultaneously fitted using the software NONMEM ${ }^{\mathrm{TM}}$. Data were best described using a two-compartment model with the parameters central $\left(V_{1}\right)$ and peripheral distribution volume $\left(V_{2}\right)$, intercompartmental $(Q)$ and linear $(C L L)$ clearance and an additional nonlinear elimination pathway $\left(K_{m}, V_{\text {max }}\right)$. Structural parameters were in agreement with immunoglobulin characteristics. In total, interindividual variability on $V_{\max }, C L L, V_{1}$ and $V_{2}$ and interoccasion variability on CLL was $22-62 \%$ CV. A covariate analysis identified weight having an influence on $V_{1}(+0.44 \%$ per $\mathrm{kg})$ and $\mathrm{CLL}(+0.87 \% \mathrm{per} \mathrm{kg})$. All parameters were estimated with good precision (RSE<39\%). A robust population pharmacokinetic model for matuzumab was developed, including a nonlinear pharmacokinetic process. In addition, relevant and plausible covariates were identified and incorporated into the model. When correlated to efficacy, this model could serve as a tool to guide dose selection for this 'targeted' cancer therapy.
\end{abstract}

British Journal of Cancer (2008) 98, 900-906. doi:10.1038/sj.bjc.6604265 www.bjcancer.com

Published online 4 March 2008

(c) 2008 Cancer Research UK

Keywords: matuzumab; population pharmacokinetic; monoclonal antibody; linear and nonlinear clearance

Recent discoveries in biomolecular processes in tumour growth led to the evolution of new targeted therapies in cancer treatment (Ross et al, 2003). Monoclonal antibodies as one of these new potential therapeutic agents benefit from their ability to bind to specific structural targets leading to good clinical efficacy and a general lower incidence of adverse events than small molecule therapeutics (Stern and Herrmann, 2005).

Matuzumab is a humanised recombinant monoclonal antibody $(\mathrm{mAb})$ of the immunoglobulin subclass IgG1 ( $\kappa$-chain) targeting the epidermal growth factor receptor (EGFR, HER1 and c-ErbB-1) (Kollmannsberger et al, 2006). This physiological transmembrane receptor with protein tyrosine kinase activity is activated by natural ligands such as epidermal growth factor and transforming growth factor- $\alpha$ (Watanabe et al, 1994; Wells, 1999; Harari, 2004). The EGFR is constitutively expressed in many healthy epithelial tissues, including skin and hair follicle. It is overexpressed or upregulated in a variety of tumour entities (e.g. colon, mamma and

*Correspondence: Professor Dr C Kloft, Department of Clinical Pharmacy, Institute of Pharmacy, Martin-Luther-Universitaet Halle-Wittenberg, Wolfgang-Langenbeck Strasse 4, 06120 Halle, Germany; E-mail: charlotte.kloft@pharmazie.uni-halle.de

Received 13 December 2007; accepted 17 January 2008; published online 4 March 2008 bronchial carcinoma) and is often associated with a high metastatic rate, poor prognosis and advanced disease progression (Dassonville et al, 1993; Rusch et al, 1993; Salomon et al, 1995). Epidermal growth factor receptor can be used as a target for therapies based on blockade of receptor-ligand interactions and inhibition of downstream signalling pathways such as cell proliferation, angiogenesis and invasion as well as increase of apoptosis (Ritter and Arteaga, 2003; Li et al, 2005). Epidermal growth factor receptor and other members of this receptor family have already been successful targets for cancer therapy (Wells, 1999).

The history of targeting the EGFR with matuzumab started with the murine mAb 425 generated by immunisation of mice (Murthy et al, 1987). From a subclone, the antibody EMD55900 was produced causing immune reactions in patients by developing human anti-mouse antibodies.

The first approved $\mathrm{mAb}$ targeting EGFR as a single agent or in combination with irinotecan in patients with EGFR-expressing, metastatic and irinotecan-refractory colorectal carcinoma is the chimeric mAb cetuximab (Harding and Burtness, 2005), which has shown favourable efficacy (Cunningham et al, 2004).

The most common adverse reaction was reported to be skin toxicity, including acneform rash. To overcome the disadvantages of the murine or chimeric mAbs and to not or only marginally induce anti-antibodies and cause an immune response, the 
humanisation of the murine mAb 425 was performed, according to the method of G. Winter and as described by Kettleborough et al (1991). The humanised mAb matuzumab has already shown promising activity in several phase I and phase II studies in the treatment of different tumour entities (Vanhoefer et al, 2004).

The overall aim of this population analysis was to develop a PK model comprehensively characterising the pharmacokinetics of matuzumab. An accurate description of the typical PK profile and of different variability types over a broad range of dosing regimens was to be provided. In addition, patient-specific characteristics should be attempted to be identified to explain the variability of the PK parameters and to further guide dosage regimen decisions for following trials.

\section{MATERIALS AND METHODS}

\section{Patient population, treatment and data collection}

This study included 90 patients (53 males and 37 females) from three phase I, open labelled, nonrandomised, uncontrolled, multicentre studies. The patients had different types of advanced carcinoma. The main criteria for inclusion encompassed histologically proven carcinoma in advanced, nonresectable and/or metastatic state, Karnofsky performance status $\geqslant 60 \%$, life expectancy $>12$ weeks ( 1 study $>8$ weeks), no prior chemo- or radiotherapy within the last 3 or 4 weeks prior to study start, adequate renal, haematological and hepatic function, age $\geqslant 18$ years. Patients were excluded if they had known brain metastases, were pregnant or had other relevant medical criteria. All patients gave written informed consent prior to inclusion into the study. The clinical studies were carried out after approval from ethics committee.

Patients received matuzumab as multiple $1 \mathrm{~h}$ i.v. infusions in a constant dosing regimen. A wide range of dosing regimens was covered by different groups of patients. In study 1 , matuzumab was administered starting with $400 \mathrm{mg}$ weekly, followed by $800 \mathrm{mg}$ biweekly and up to $800 \mathrm{mg}$ every week in combination with a fixed dose of gemcitabine $\left(1000 \mathrm{mg} \mathrm{m}^{-2}\right)$. Study 2 included the administration of $400,800,1200$ and $1600 \mathrm{mg}$ with a 3-week interval and, additionally, $1200 \mathrm{mg}$ biweekly and weekly administration. In study 3, matuzumab was given $400,800,1200$ or $1600 \mathrm{mg}$ once weekly. Some patients were treated over a longer time period of approximately 1 year.

Serum samples were taken pre- and post-infusions. Frequent serum samples were taken after the first infusion and after the infusion given 3 weeks later. Before and after several other infusions peak and trough concentrations were analysed. Details of dose regimens and sampling schedules are presented in Table 1. Frozen samples were shipped to the Institute of Drug Metabolism and Pharmacokinetics, Merck KGaA, Grafing, Germany, for bioanalysis. Serum levels were evaluated using a validated sandwich enzyme-linked immunosorbent assay method, as described previously (Vanhoefer et al, 2004). Precision and accuracy met the international recommendations for bioanalytical immunoassays (DeSilva et al, 2003).

\section{Population pharmacokinetic analysis}

The population PK analysis was performed with serum mAb concentration data and associated data such as demographics from all three studies. The population PK model building process was performed using the nonlinear mixed-effects modelling approach implemented in the software NONMEM $^{\mathrm{TM}}$, version V, level 1.1. Model development was performed stepwise. First a structural model was developed including an investigation of different compartment models. During model refinement, linear and nonlinear processes and elimination pathways were included.

During the development of the base model and at later stages, interindividual, residual and interoccasion variabilities were investigated. As part of the statistical submodel, the interindividual variability (IIV) was modelled with an exponential random effects term:

$$
P_{\mathrm{ki}}=\theta_{\mathrm{k}} \cdot \mathrm{e}^{\eta_{\mathrm{ki}}}
$$

where $P_{\mathrm{ki}}$ represents the parameter value $k$ from the individual i and $\theta_{\mathrm{k}}$ describes the population value of the parameter $k . \eta_{\mathrm{ki}}$ denotes the ln-difference between $P_{\mathrm{ki}}$ and $\theta_{\mathrm{k}}$. The interoccasion variability (IOV), that is the variability within one individual between study occasions, was also examined with an exponential random effects term:

$$
P_{\text {kiq }}=\theta_{\mathrm{k}} \cdot \mathrm{e}^{\eta_{\mathrm{ki}}+\kappa_{\mathrm{kiq}}}
$$

where $P_{\text {kiq }}$ is the individual parameter value $k$ from the individual i at the occasion $\mathrm{q}$ that differs from the typical individual value by an additional random effect $\kappa_{\text {kiq }}$. An occasion was characterised as the time period from the start of an infusion and until the start of the next administration. $\eta_{\mathrm{ki}}$ and $\kappa_{\mathrm{kiq}}$ were assumed to be symmetrically distributed with a zero mean and a variance of $\omega^{2}$ and $\pi^{2}$, respectively.

Residual variability represents the discrepancy between the observed and the model-predicted concentrations after incorporation of IIV and IOV. It was modelled using additive, proportional or combined error models.

All models were parameterised in terms of clearance(s) and volume(s) using the subroutine ADVAN6 TRANS1 TOL5 in NONMEM. The analyses were performed using different estimation methods (first-order, FO; first-order conditional estimation, FOCE, with or without interaction). For the final model, FOCE with interaction was used.

\section{Covariate model building}

To advance the population analysis, a covariate analysis was performed starting from the base model. The analysis included two sequential methods to investigate whether covariates could explain part of the variability of the PK parameters: the GAM analysis

\begin{tabular}{|c|c|c|c|c|}
\hline Study & Tumour entity & Dose regimen & Planned sampling times ${ }^{a}$ & $\begin{array}{l}\text { No. of } \\
\text { subjects }\end{array}$ \\
\hline I & $\begin{array}{l}\text { Advanced pancreatic } \\
\text { cancer }\end{array}$ & 400 mg q lw; 800 mg q2w; 800 mg qlw & Baseline, I, 2, 5, 48, 96, 168, 672, 673, 674, 677, 720, 840 b & 17 \\
\hline 2 & $\begin{array}{l}\text { Various advanced cancer } \\
\text { (mainly colon/rectum } \\
\text { cancer) }\end{array}$ & $\begin{array}{l}1200 \mathrm{mg} \text { q Iw, q2w, q3w; } 400 \mathrm{mg} \text { q3w; } 800 \mathrm{mg} \\
\text { q3w; I } 600 \mathrm{mg} \text { q3w }\end{array}$ & $\begin{array}{l}\text { For q I w: baseline, I, 2, 5, 48, 96, I68 } \text {; for q2w: baseline, I, } \\
2,5,48,96,168,336^{\text {b}} \text {; for q3w: baseline, I, 2, 5, 48, I68, } \\
336,504,505,506,509,552,672,840,1008^{\text {b }}\end{array}$ & 51 \\
\hline 3 & $\begin{array}{l}\text { Various advanced cancer } \\
\text { (mainly colon/rectum } \\
\text { cancer) }\end{array}$ & $\begin{array}{l}400 \mathrm{mg} \text { q I w; } 800 \mathrm{mg} \text { q I w; } 1200 \mathrm{mg} \text { q Iw; } \\
1600 \mathrm{mg} \text { q I w; } 2000 \mathrm{mg} \text { q Iw (from week2: } \\
1600 \mathrm{mg} \text { ) }\end{array}$ & $\begin{array}{l}\text { Baseline, } 1,2,5,24,72,96,168,504,505,506,509,528 \\
576,600,672^{b}\end{array}$ & 22 \\
\hline
\end{tabular}

Table I Study characteristics with matuzumab given as multiple I h i.v. infusions ranging from 400 to $2000 \mathrm{mg}$, q I w-q3w

${ }^{a}$ Relative time elapsed after start of first infusion, in hours. ${ }^{b}$ Before and after several other infusions peak and trough concentrations were analysed. 
('generalised additive modelling', implemented in the software Xpose $^{\mathbb{R}}$ ), using the Akaike's Information Criterion (Jonsson and Karlsson, 1999; Pan, 2001), and the covariate analysis within NONMEM. The GAM analysis allowed a fast initial screening of covariates (Mandema et al, 1992), which were retrieved by the insertion of covariate relations into the NONMEM model. In NONMEM, all covariate relations were investigated by forward inclusion and backward deletion techniques. The final covariate model was built in a stepwise manner. In each step, all possible/ remaining parameter-covariate relations were assessed. The included covariate relation that led to the largest drop in the objective function value (OFV), the criterion for model evaluation, was kept in the model. In the next steps, the remaining covariate relations were investigated. All covariate relations that caused a $\Delta$ OFV $>-3.84(P<0.05, \mathrm{df}=1)$ formed the full covariate model. From the full model, the covariate relations were then deleted one at a time using a stricter criterion $(\Delta \mathrm{OFV}>10.83, P<0.001$, $\mathrm{df}=1$ ). The final covariate model was achieved when deletion of each covariate relation was significant.

Covariates investigated for their influence on PK parameters included continuous characteristics as demographics (weight (WT), height (HT), age, body surface area (BSA), body mass index (BMI)), laboratory values (creatinine clearance (CLCR), lactate dehydrogenase (LDH), alkaline phosphatase (AP), white blood cell count (WBC)) and others (Karnofsky index, dose group (DSG)), as well as the categorical characteristics (sex, study number, study site (SID) and concomitant chemotherapy (COME)).

Continuous covariates were investigated with a linear covariate model:

$$
\mathrm{TVP}_{\mathrm{k}}=\theta_{\mathrm{k}} \cdot\left(1+\theta_{\mathrm{COV}} \cdot\left(\mathrm{COV}-\mathrm{COV}_{\text {median }}\right)\right)
$$

where $\operatorname{TVP}_{\mathrm{k}}$ is the population value of the parameter $P_{\mathrm{k}}$ for a specific covariate value (COV) and $\theta_{\mathrm{k}}$ is the population value of the parameter $P_{\mathrm{k}}$ with the covariate value being the median value $\left(\mathrm{COV}_{\text {median }}\right) . \theta_{\text {cov }}$ is the fractional change in the population parameter with each unit change from the median covariate value. Whether graphical inspection suggested, nonlinear relations were investigated with a power model, an $E_{\max }$ model or an exponential model.

Categorical covariates were given as dichotomous variables (sex: male/female, concomitant chemotherapy: yes/no). The coding will be illustrated using an indicator variable (IND), being 0 or 1 (e.g. male or female).

$$
\mathrm{TVP}_{\mathrm{k}}=\theta_{\mathrm{k}} \cdot\left(1+\theta_{\mathrm{COV}} \cdot(\mathrm{IND})\right)
$$

where $\theta_{\mathrm{k}}$ is the typical value of the parameter $P_{\mathrm{k}}$ when IND $=0$. $\theta_{\text {cov }}$ represents the fractional increase or decrease of the parameter $P_{\mathrm{k}}$ caused by IND $=1$.
If the categorical covariate had multiple categories, each category had an IND (e.g. study site: site $1=(\mathrm{IND}) 1$, etc.).

$$
\operatorname{TVP}_{\mathrm{k} 1}=\theta_{\mathrm{k} 1} \quad \text { if }(\mathrm{IND})=1 \text {, etc }
$$

where $\theta_{\mathrm{kx}}$ is the typical value of the parameter $P_{\mathrm{k}}$ for each category $_{\mathrm{x}}$.

Visual exploratory analysis of the covariate relations revealed that for the laboratory parameters aspartate aminotransferase, alanine aminotransferase, gamma glutamyltransferase and bilirubin, a 'relation' was driven by very few individuals. Neglecting these individuals did not support the relation anymore. Consequently, these relations were not considered in further covariate analysis.

\section{RESULTS}

\section{Patient population}

Patient characteristics with descriptive statistics are presented in Table 2. For all parameters, the range was wide, for example, age was varying from 29 to 82 years, which was beneficial for the ability to identify covariate relations. The table also includes the number of the missing values that were replaced for the modelbuilding process by the respective median value.

\section{Base model}

A total of 1256 serum concentrations from 0.258 to $1157 \mu \mathrm{g} \mathrm{ml}^{-1}$ were simultaneously analysed. The average number of concentrations per patient was 15 (range: 5-24). Serum concentration-time profiles were best described by a two-compartment model (e.g. $\Delta \mathrm{OFV}>-300$ compared with a one-compartment model, $P<0.05)$. Within this model in addition to the linear clearance (CLL), a second elimination pathway as a nonlinear process (Michaelis-Menten kinetics, CLNL) from the central compartment was included with the additional parameters $V_{\max }$, the maximum elimination rate $\left(\mathrm{mg} \mathrm{h}^{-1}\right)$, and $K_{\mathrm{m}}$, the concentration $\left(\mu \mathrm{g} \mathrm{ml}^{-1}\right)$ with half-maximal elimination rate $(\Delta \mathrm{OFV}>-100)$. A need to incorporate nonlinearity might also be concluded from the semilogarithmic plots in Figure 1, showing the geometric mean and the standard deviation of the observed concentration-time profiles of four weekly dose regimens of $400-1600 \mathrm{mg}$, after the first and fourth infusion. In the terminal phase, the slope of the curve was steeper at lower concentrations.

The final base structural model is shown in Figure 2. In this model, the input was the route of administration (i.v. infusion). The central compartment with the central volume of distribution $\left(V_{1}\right)$ was linked with the peripheral

\begin{tabular}{|c|c|c|c|c|c|}
\hline & Study I & Study 2 & Study 3 & Total & Missings \\
\hline Number of IDs, (male/female) & $17(9 / 8)$ & $51(33 / 18)$ & $22(|| /||)$ & $90(53 / 37)$ & 0 \\
\hline Age (years), median (min-max.) & $65(40-82)$ & $57(29-78)$ & $58(30-71)$ & $60(29-82)$ & 0 \\
\hline Height (cm), median (min-max) & $168(156-183)$ & $169(143-198)$ & $170(150-184)$ & $169(143-198)$ & 3 \\
\hline Weight $(\mathrm{kg})$, median $(\min -\max )$ & $68(48-81)$ & $7 \mid(47-125)$ & $72(44-98)$ & $7 \mid(44-125)$ & 3 \\
\hline Body mass index $\left(\mathrm{kg} \mathrm{m}^{-2}\right)$, median (min-max) & $24.7(17.0-30.7)$ & $25.8(20.1-37.0)$ & $24.3(15.9-33.9)$ & $24.9(15.9-37.0)$ & 4 \\
\hline Body surface area $\left(\mathrm{m}^{2}\right)$, median $(\min -\max )$ & $1.77(|.5|-2.0 \mid)$ & $1.82(1.34-2.59)$ & $1.85(1.44-2.16)$ & $1.82(1.34-2.59)$ & 4 \\
\hline Creatinine clearance $\left(\mathrm{ml} \mathrm{min}{ }^{-1}\right)$, median $(\min -\max )$ & $104\left(7 \mid-480^{a}\right)$ & $83(47-180)$ & $108(47-226)$ & $91\left(41-480^{a}\right)$ & 3 \\
\hline Alkaline phosphatase $\left(\cup I^{-1}\right)$, median $(\min -\max )$ & $190(118-1026)$ & $156(40-403)$ & $235(96-1309)$ & $17 \mid(40-1309)$ & 0 \\
\hline Lactate dehydrogenase $\left(\cup I^{-1}\right)$, median (min-max) & $171(117-926)$ & $542(305-8041)$ & $187(76-3243)$ & $426(76-8041)$ & 0 \\
\hline Study centre number (number of IDs) & $\begin{array}{cl}1 & 2 \\
(8) & (9)\end{array}$ & $\begin{array}{l}3 \\
(5 \mathrm{I})\end{array}$ & $\begin{array}{c}4 \\
(22)\end{array}$ & $\begin{array}{cccc}1 & 2 & 3 & 4 \\
(8) & (9) & (51) & (22)\end{array}$ & 0 \\
\hline Combination chemotherapy, number of IDs & 17 & 0 & 0 & 17 & 0 \\
\hline
\end{tabular}
compartment with the peripheral volume of distribution $\left(V_{2}\right)$ via

Table 2 Characteristics of the study population (ID = subject) including number or median, range and number of missings

${ }^{a}$ Spurious value in original data set, but without influence on population pharmacokinetic analysis. 

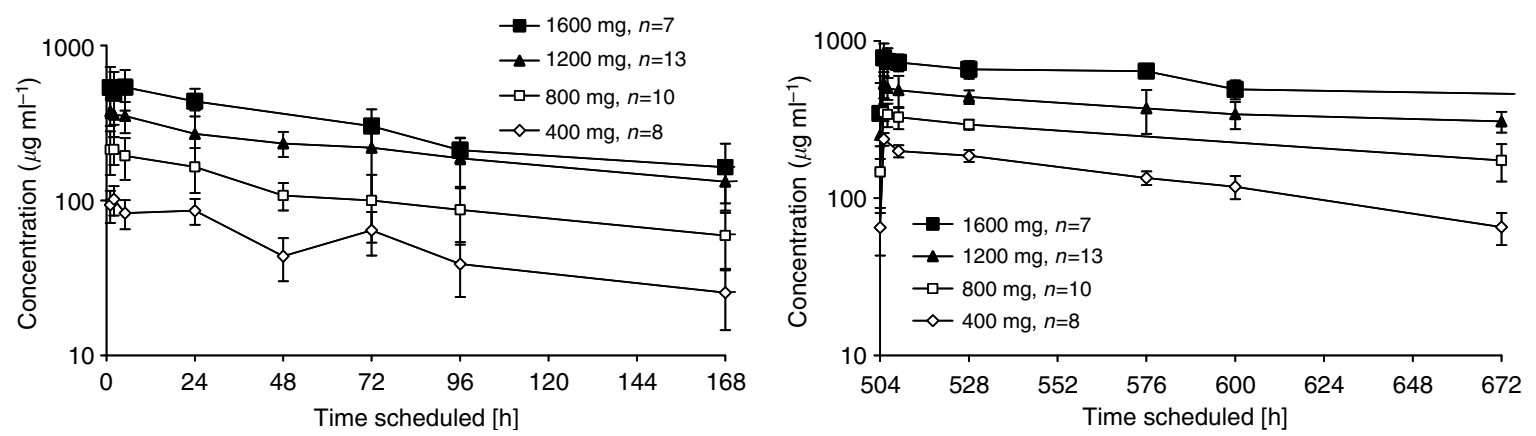

Figure I Semilogarithmic plot of the geometric mean and the standard deviation of the observed concentration-time profiles of the four weekly dose regimens (400, 800, I 200 and $1600 \mathrm{mg}$ per week) after the first (left panel) and fourth (right panel) infusion. $n=$ number of patients in the dose group. Last time point for $1600 \mathrm{mg}$ dose group was after $1008 \mathrm{~h}$ and is not shown.

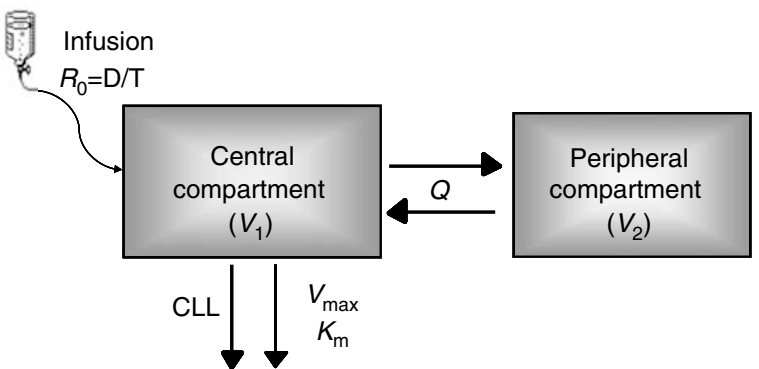

Figure 2 Schematic structural pharmacokinetic model. $R_{0}=$ infusion rate; $D=$ dose; $T=$ infusion duration; $V_{1}=$ volume in the central compartment; $Q=$ intercompartmental clearance; $V_{2}=$ volume in the peripheral compartment; $C L L=$ linear clearance part; $V_{\max }=$ maximum elimination rate; $K_{m}=$ concentration at which the elimination rate is $50 \%$ of the maximum value.

$Q$, the intercompartmental clearance. Implementation of CLNL from the peripheral compartment only or from both compartments did not result in an improvement of the model. The linear part of clearance was estimated to be $14.6 \mathrm{ml} \mathrm{h}^{-1}$ and the nonlinear part, calculated from the parameters $K_{\mathrm{m}}\left(5.3 \mathrm{mgl}^{-1}\right)$ and $V_{\max }$ $\left(0.552 \mathrm{mg} \mathrm{h}^{-1}\right)$ at $\mathrm{mAb}$ concentrations $\ll K_{\mathrm{m}}$, to be $104.2 \mathrm{ml} \mathrm{h}^{-1}$, respectively.

Total clearance as the sum of CLL and CLNL was $118.8 \mathrm{ml} \mathrm{h}^{-1}$ (at mAb concentrations $\ll K_{\mathrm{m}}$ ). In Figure 3 left panel, the dependence of total clearance on the concentration of the $\mathrm{mAb}$ is presented. At low $\mathrm{mAb}$ concentrations, until approximately $1 \mu \mathrm{g} \mathrm{ml}^{-1}$, total clearance (solid line) was mainly influenced by the nonlinear clearance part (long-dashed line). At higher mAb concentrations, the impact of the nonlinear part on the total clearance decreased and the linear part (short-dashed line) was dominating. In accordance with the nonlinear behaviour, the halflife ranged between 4.4 and 10.5 days at concentrations of 20 and $1000 \mu \mathrm{g} \mathrm{ml}^{-1}$, respectively (Figure 3, right panel).

Interindividual variability was quantified for CLL, $V_{1}, V_{2}$ and $V_{\max }$, and in addition, IOV was implemented. The inclusion of IOV was limited to eight infusions due to insufficient data hereafter and implemented by different ways of assigning the eight infusions to a varying number of occasions. The best result (lowest $\mathrm{OFV}$, smallest relative standard errors in \% $(\mathrm{RSE}=$ standard error divided by population estimate $\left.{ }^{\star} 100\right)$ ) was achieved with IOV on CLL, where every infusion corresponded to one occasion $(\triangle \mathrm{OFV} \sim-400)$.

In general, in the base model, IIV was moderate $(24-60 \% \mathrm{CV})$ and larger than IOV of $23 \% \mathrm{CV}$. Residual variability was best implemented with a combined error model with a proportional error of $13 \% \mathrm{CV}$ and an additive error of $0.312 \mathrm{mg} \mathrm{l}^{-1}$, being fixed due to model stability (the value was chosen from prior plausible successfully run models).

The parameters obtained from the base model are shown in Table 3, left part, including the RSE. All parameters were generally estimated with good precision (RSE $\leqslant 37.1 \%$, except for IIV on CLL and $\left.V_{\max } \leqslant 49.4 \%\right)$.

\section{Final model}

Fourteen covariate relations were found by GAM: $V_{\max } \sim \mathrm{BSA}$, sex, CLCR; $V_{1} \sim \mathrm{BSA}$, COME, sex; $V_{2} \sim \mathrm{COME}$, sex, LDH, SID; $\mathrm{CLL} \sim \mathrm{BSA}, \mathrm{COME}, \mathrm{AP}$, sex.

These screening results were included into the NONMEM base model. After forward inclusion, the full covariate model contained eight relations: $V_{\max } \sim \mathrm{BSA} ; V_{1} \sim \mathrm{WT}$, DSG, COME; $V_{2} \sim \mathrm{BSA}, \mathrm{DSG}$, COME; CLL $\sim \mathrm{BSA}(\triangle \mathrm{OFV}$ in total: -102). After backward elimination, the remaining statistically significant covariate relations were $V_{1} \sim \mathrm{WT}, \mathrm{DSG}, V_{2} \sim \mathrm{COME}$ and $\mathrm{CLL} \sim \mathrm{BSA}$. The OFV compared with the base model was reduced by 69 .

The significant relations identified by NONMEM were further examined for plausibility and relevance. Body size measures were identified to be plausible explanatory factors for the PK parameters $V_{1}$ and CLL. Regarding the influence of DSG on $V_{1}$, the graphical inspection indicated that the relation was driven by very few patients only in the highest dose group. In addition, the influence of the dose group was inspected by simulation of 'dummy' patients of the lowest-, median- and highest-dose group. As the resulting concentration-time profiles were very similar, this covariate relation was removed from the model. An analogous procedure was performed for the covariate COME on CLL. No major difference was found, and, as additionally, no biologically plausible explanation can be given for this covariate relation, it was also removed from the model.

During covariate modelling, IIV on $V_{\max }$ decreased from the base to the final covariate model, although no covariate on $V_{\max }$ was included. Therefore, different correlations were investigated revealing correlations between $V_{\max }, V_{1}$ and $V_{2}$. The correlation coefficients are given in Table 3 (right panel). After the inclusion of the correlations, the model contained the remaining covariates WT on $V_{1}$ and BSA on CLL. As BSA and WT were highly correlated, it was examined whether the covariate BSA could be exchanged by WT. It was shown that the model with WT on CLL was not inferior to the model with BSA on CLL. From the individual plots (observed and predicted concentrations $v s$ time), no difference between the two covariate models was seen. Additionally, compared with WT, BSA displayed a relatively small range of values, and especially there were only few study patients with high BSA values. Another criterion to support the replacement of the covariates was given by the inspection of the distribution of the individual CLL. The model with WT on CLL better followed a 

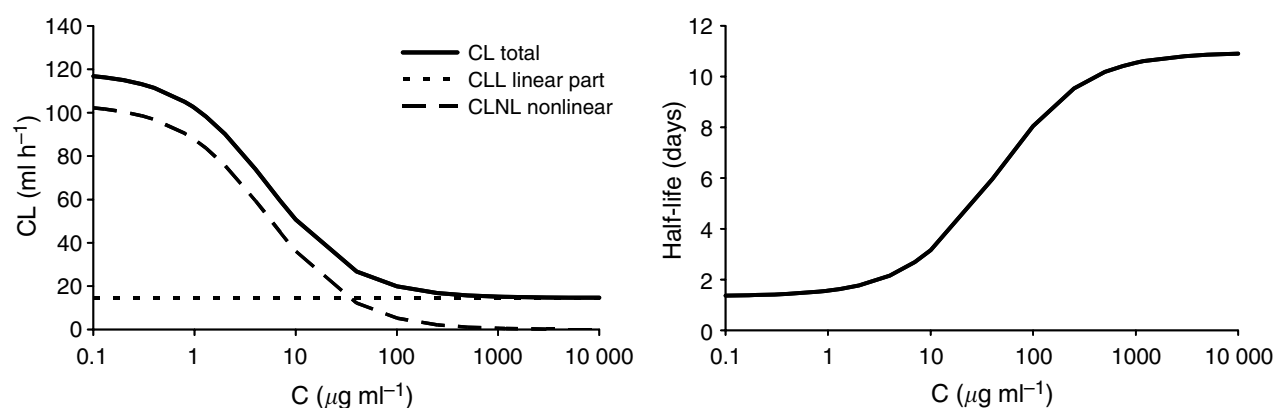

Figure 3 Left panel: dependence of clearance on concentration $(C)$ of the mAb. Right panel: dependence of half-life (in days) on $C$.

Table 3 Population pharmacokinetic estimates of matuzumab obtained from the base and the final model

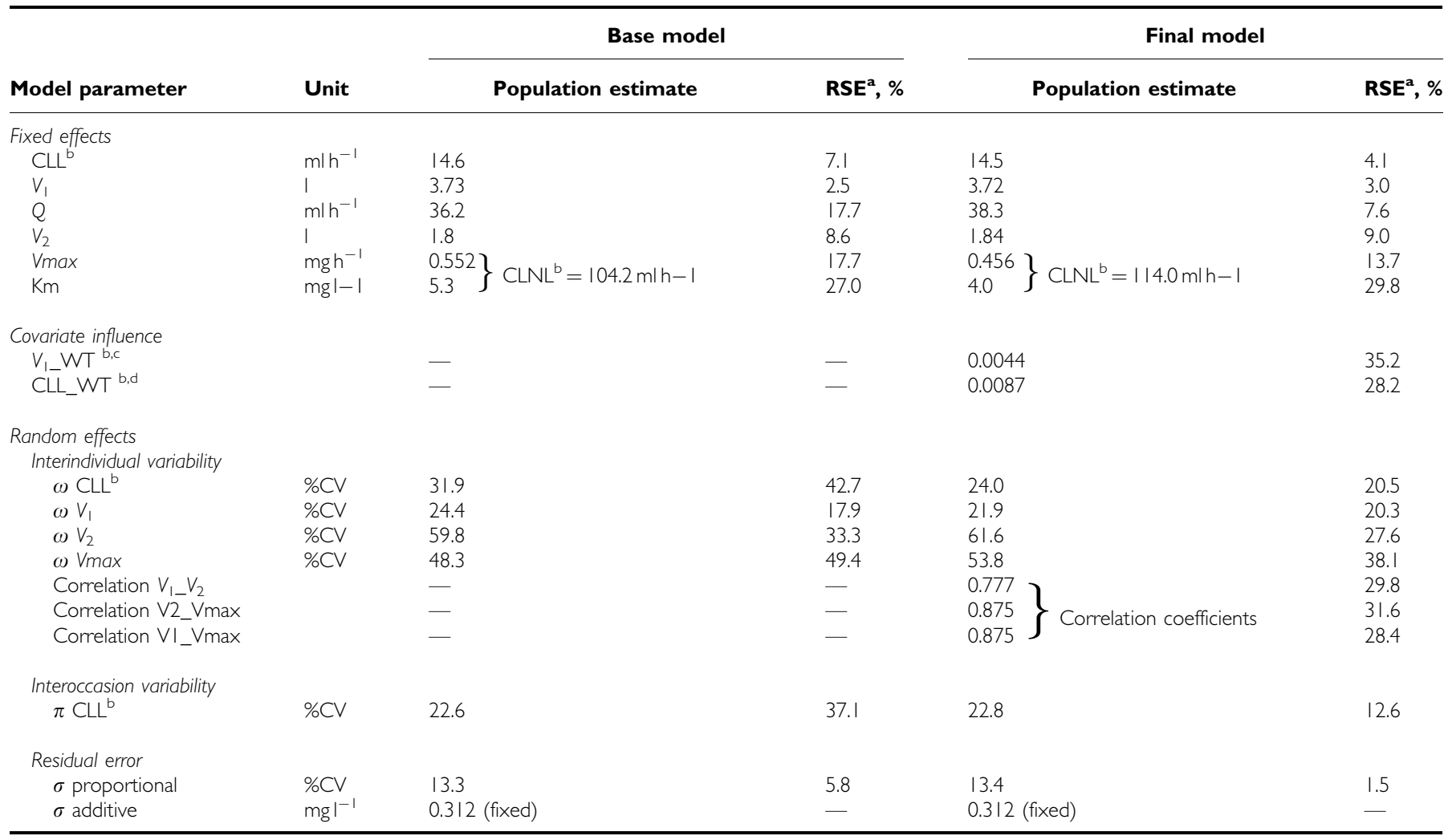

${ }^{a}$ Relative standard error (standard error divided by population estimate* 100 ; for the random effects parameters RSE is related to the corresponding variance scale). ${ }^{b} \mathrm{CLL}$ : linear clearance part; CLNL: nonlinear clearance part (at concentrations $\ll K m$ ); $W T$ : weight. ${ }^{c} V_{1, \text { individual }}=V_{1} *\left[I+V_{1}-W T *\left(W_{T}-W T_{\text {median }}\right)\right]^{*} E X P\left(\eta V_{1}\right)$. ${ }^{\mathrm{d} C L L_{\text {individual }}}=\mathrm{CLL} *\left[\mathrm{I}+\mathrm{CLL}-\mathrm{WT} *\left(\mathrm{WT}-\mathrm{WT} \mathrm{T}_{\text {median }}\right)\right] * \mathrm{EXP}(\eta \mathrm{CLL}+\kappa \mathrm{CLL})$.

normal distribution pattern. The exchange of the covariates caused an increase in the OFV by only 3.4. As WT is a directly measured variable in the daily clinical process compared with the derived variable BSA, and because, in summary, it was demonstrated that similar results were obtained from the covariate exchange, the final model included WT on CLL instead of BSA, besides WT on $V_{1}$. A deviation of $-19 \%$ to $+25 \%$ of the population PK parameters $\left(V_{1}\right.$, CLL) for the 5th and 95th percentile of the WT values in the study population with respect to the population PK parameters for the median WT value was observed.

All parameter estimates obtained from the final model are shown in Table 3 (right part). $V_{1}, V_{2}$, CLL, $Q, V_{\max }$ and $K_{\mathrm{m}}$ of the final model were similar to the estimates of the base model. IIV on CLL and $V_{1}$ decreased by 25 and $10 \%$, respectively. In general, all parameters were estimated with a better precision than in the base model (RSE $<39 \%)$. Especially for the IIV (except on $V_{1}$ ) and IOV, more precise estimates were obtained.
In Figure 4, the goodness-of-fit plots obtained from the final population PK model in linear (left) and logarithmic (right) scale are shown. The upper panel presents the population predictions $v s$ the observed concentrations. Especially the data points in the low region were uniformly spread around the line of unity with a slight underprediction in the higher region. Examining the lower panel with individual predicted $v s$ observed concentrations, those in the higher region were more uniformly scattered, and the lower concentrations were closer to the line of unity. Overall, the plots indicate that the study data were sufficiently well described by the developed model.

\section{DISCUSSION AND CONCLUSION}

In this study, a population PK analysis was performed for the humanised $\mathrm{mAb}$ matuzumab directed against the EGFR with data 

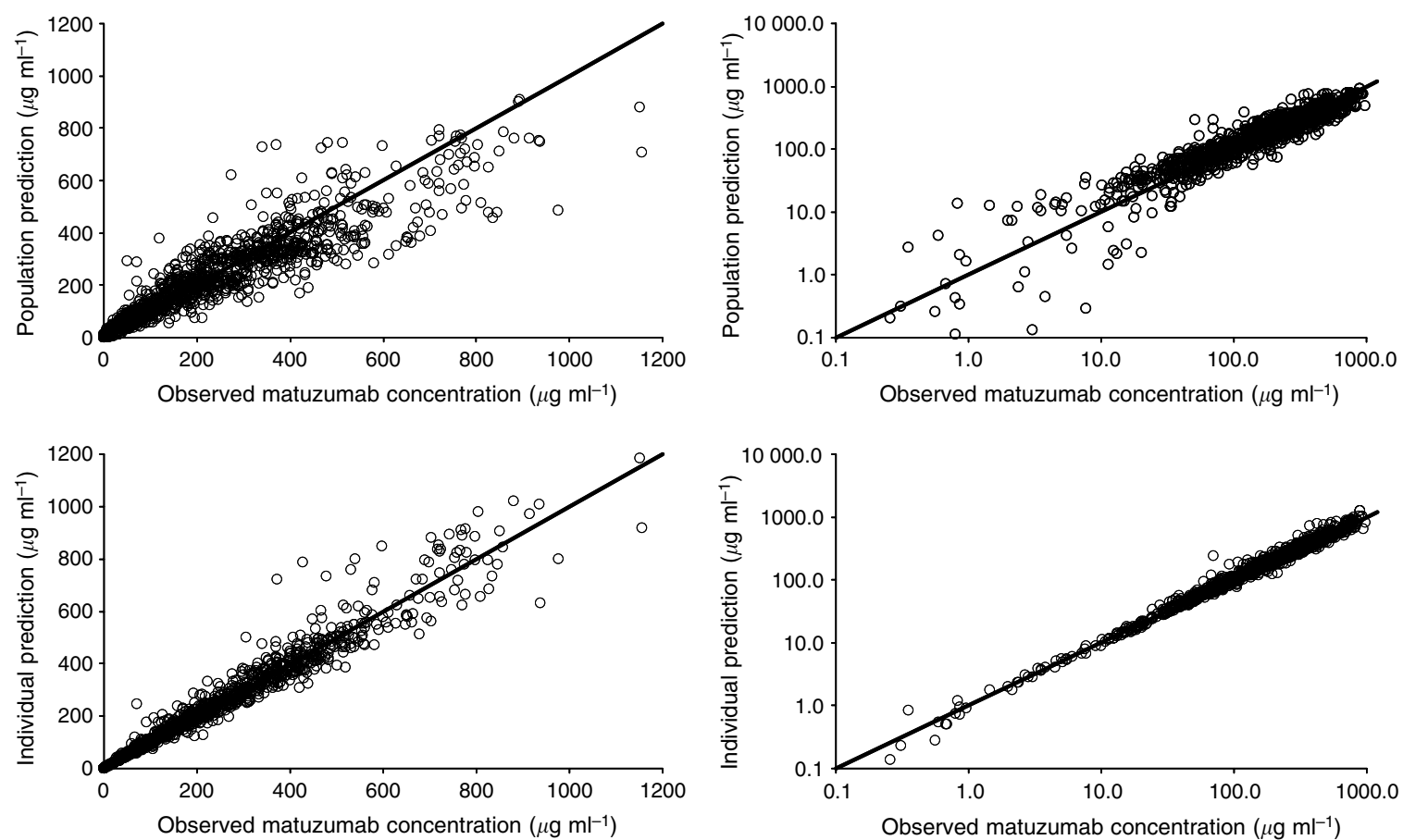

Figure 4 Goodness-of-fit plots. Population predictions (upper panel) and individual predictions (lower panel) vs observed matuzumab serum concentrations are shown using linear (left) and logarithmic (right) scale of both axes.

from three phase I studies. The model was developed using over 1200 serum concentration data points from 90 cancer patients with widely differing characteristics and multiple dosing regimens.

The structural model comprised two compartments with two elimination pathways from the central compartment, one linear and one nonlinear (Michaelis-Menten). Nonlinear PK behaviour has also been reported for other mAbs, such as sibrotuzumab and clenoliximab (Mould et al, 1999; Kloft et al, 2004). It has also been investigated in addition to the linear elimination for rituximab, but the model did not perform significantly better than the simple linear model. However, goodness-of-fit plots suggest a misspecification for high concentrations ( $\mathrm{Ng}$ et al, 2005).

With respect to the structural model, matuzumab was initially distributed to a restricetd central volume of distribution of 3.71 and an even smaller peripheral volume of distribution of $1.8 \mathrm{l}$, which indicated that matuzumab was not (largely) distributed apart from serum volume. Beside the low peripheral volume, the intercompartmental clearance $Q$ also indicated a limited distribution, which was consistent with the behaviour of endogenous IgG immunoglobulins (Morell et al, 1970; Koleba and Ensom, 2006; Kuester and Kloft, 2006). In total, matuzumab showed similar PK characteristics (clearance and volumes of distribution) to other therapeutic mAbs following intravenous administration (Mould et al, 1999; Kovarik et al, 2001; Bruno et al, 2005).

Three components of random variability (interindividual, interoccasional and residual) were implemented into the matuzumab model. With the relatively small residual variability $(13.4 \%$ $\mathrm{CV}$ for the proportional part and a fixed additive error of $0.312 \mathrm{mg} \mathrm{l}^{-1}$ ), it can be suggested that the developed model possess reasonably high predictability. IOV has been rarely investigated in $\mathrm{mAb}$ research, but in more recent population $\mathrm{PK}$ analysis, it has been included to improve the model (Kloft et al, 2004; Fang et al, 2007). The estimated IOV of matuzumab (23\% CV; RSE, $13 \%)$ was in the range or slightly higher than that for other immunologicals: sibrotuzumab, humanised antibody $\mathrm{HuCC} 49 \mathrm{CH} 2$ and etanercept showed 13,11 and $28 \% \mathrm{CV}$, as well as RSE imprecisions of 25 and
$102 \%$ (not reported for etanercept), respectively (Lee et al, 2003; Kloft et al, 2004; Fang et al, 2007). The importance of implementing IOV in population PK analysis has been demonstrated (Karlsson and Sheiner, 1993) and investigation of IOV avoids biased population parameter estimates.

The aim of building the covariate model was to find patient- or study-specific characteristics, which could explain and thus reduce the variability of the base model. The inclusion of the covariates WT on $V_{1}$ and WT on CLL showed a significant improvement of the model, which could be seen by the reduction of the IIV on CLL by approximately $25 \%$. As no difference was observed between patients of either sex and between matuzumab as a single agent and in combination with gemcitabine, no effect of sex and gemcitabine on the PK of matuzumab might be assumed. Additionally, kidney and liver functions do not seem to influence the PK. These results are in good agreement with investigations of other mAbs. The population PK analysis for trastuzumab showed a significant influence of WT on $V_{1}$ but was not considered clinically relevant (Bruno et al, 2005). Similar results have been reported for the chimeric antibody basiliximab (Kovarik et al, 2001). The incorporation of WT on $V_{1}$ for golimumab, a fully human mAb, significantly improved the model (Zhou et al, 2007).

Future research could attempt to explain the mechanism of nonlinearity in the PK. An appealing approach might present physiologically based modelling including processes, such as receptor internalisation. It has been demonstrated that binding of a $\mathrm{mAb}$ to the neonatal $\mathrm{Fc}$ receptor $(\mathrm{FcRn})$ and internalisation of the resulting complex results in the long serum half-life of the antibody (Brambell et al, 1964; Ghetie et al, 1997). Comparing a modified therapeutic antibody (different Fc part) with its original antibody, the resulting half-life for the modified antibody was shorter. It could be assumed that the modified antibody might less be able to bind to the FcRn and thus, was less protected from elimination (Fang et al, 2007).

The overall aim of this final model could be, when correlated to PD or efficacy data, to serve as a tool to guide selection of optimal dose regimens for matuzumab, a highly promising 'targeted' 
cancer therapy. From the performed covariate analysis, it should already be recognised that the results do not suggest dose adjustments for sex, age or organ functions such as liver or kidney. For future studies, the identification of molecular tumour markers are considered as being essential prerequisites to be linked to the $\mathrm{PK}$ results in a pharmacokinetic/pharmacodynamic model. This could lead to a better prediction of the response for this new class agent and maximise the patient's benefit.

\section{REFERENCES}

Brambell FW, Hemmings WA, Morris IG (1964) A theoretical model of gamma-globulin catabolism. Nature 203: $1352-1354$

Bruno R, Washington CB, Lu JF, Lieberman G, Banken L, Klein P (2005) Population pharmacokinetics of trastuzumab in patients with HER2+ metastatic breast cancer. Cancer Chemother Pharmacol 56: $361-369$

Cunningham D, Humblet Y, Siena S, Khayat D, Bleiberg H, Santoro A, Bets D, Mueser M, Harstrick A, Verslype C, Chau I, Van Cutsem E (2004) Cetuximab monotherapy and cetuximab plus irinotecan in irinotecanrefractory metastatic colorectal cancer. $N$ Engl J Med 351: 337-345

Dassonville O, Formento JL, Francoual M, Ramaioli A, Santini J, Schneider M, Demard F, Milano G (1993) Expression of epidermal growth factor receptor and survival in upper aerodigestive tract cancer. J Clin Oncol 11: $1873-1878$

DeSilva B, Smith W, Weiner R, Kelley M, Smolec J, Lee B, Khan M, Tacey R, Hill H, Celniker A (2003) Recommendations for the bioanalytical method validation of ligand-binding assays to support pharmacokinetic assessments of macromolecules. Pharm Res 20: 1885-1900

Fang L, Holford NH, Hinkle G, Cao X, Xiao JJ, Bloomston M, Gibbs S, Saif OH, Dalton JT, Chan KK, Schlom J, Martin Jr EW, Sun D (2007) Population pharmacokinetics of humanized monoclonal antibody HuCC $49 \Delta \mathrm{CH} 2$ and murine antibody CC49 in colorectal cancer patients. J Clin Pharmacol 47: 227-237

Ghetie V, Popov S, Borvak J, Radu C, Matesoi D, Medesan C, Ober RJ, Ward ES (1997) Increasing the serum persistence of an IgG fragment by random mutagenesis. Nat Biotechnol 15: 637-640

Harari PM (2004) Epidermal growth factor receptor inhibition strategies in oncology. Endocr Relat Cancer 11: 689-708

Harding J, Burtness B (2005) Cetuximab: an epidermal growth factor receptor chemeric human-murine monoclonal antibody. Drugs Today (Barc) 41: 107-127

Jonsson EN, Karlsson MO (1999) Xpose - an S-PLUS based population pharmacokinetic/pharmacodynamic model building aid for NONMEM. Comput Methods Programs Biomed 58: 51-64

Karlsson MO, Sheiner LB (1993) The importance of modeling interoccasion variability in population pharmacokinetic analyses. J Pharmacokinet Biopharm 21: $735-750$

Kettleborough CA, Saldanha J, Heath VJ, Morrison CJ, Bendig MM (1991) Humanization of a mouse monoclonal antibody by CDR-grafting: the importance of framework residues on loop conformation. Protein Eng 4: $773-783$

Kloft C, Graefe EU, Tanswell P, Scott AM, Hofheinz R, Amelsberg A, Karlsson MO (2004) Population pharmacokinetics of sibrotuzumab, a novel therapeutic monoclonal antibody, in cancer patients. Invest New Drugs 22: $39-52$

Koleba T, Ensom MH (2006) Pharmacokinetics of intravenous immunoglobulin: a systematic review. Pharmacotherapy 26: 813-827

Kollmannsberger C, Schittenhelm M, Honecker F, Tillner J, Weber D, Oechsle K, Kanz L, Bokemeyer C (2006) A phase I study of the humanized monoclonal anti-epidermal growth factor receptor (EGFR) antibody EMD 72000 (matuzumab) in combination with paclitaxel in patients with EGFR-positive advanced non-small-cell lung cancer (NSCLC). Ann Oncol 17: 1007-1013

Kovarik JM, Nashan B, Neuhaus P, Clavien PA, Gerbeau C, Hall ML, Korn A (2001) A population pharmacokinetic screen to identify demographicclinical covariates of basiliximab in liver transplantation. Clin Pharmacol Ther 69: $201-209$

Kuester K, Kloft C (2006) Pharmacokinetics of monoclonal antibodies. In Pharmacokinetics and Pharmacodynamics of Biotech Drugs Meibohm B (ed) pp 45-91. Wiley-VCH Verlag: Weinheim

Lee H, Kimko HC, Rogge M, Wang D, Nestorov I, Peck CC (2003) Population pharmacokinetic and pharmacodynamic modeling of etanercept using logistic regression analysis. Clin Pharmacol Ther 73: $348-365$

Li Z, Zhao R, Wu X, Sun Y, Yao M, Li J, Xu Y, Gu J (2005) Identification and characterization of a novel peptide ligand of epidermal growth factor receptor for targeted delivery of therapeutics. FASEB J 19: 1978 - 1985

Mandema JW, Verotta D, Sheiner LB (1992) Building population pharmacokinetic - pharmacodynamic models. I. Models for covariate effects. J Pharmacokinet Biopharm 20: 511-528

Morell A, Terry WD, Waldmann TA (1970) Metabolic properties of IgG subclasses in man. $J$ Clin Invest 49: 673-680

Mould DR, Davis CB, Minthorn EA, Kwok DC, Elliott MJ, Luggen ME, Totoritis MC (1999) A population pharmacokinetic-pharmacodynamic analysis of single doses of clenoliximab in patients with rheumatoid arthritis. Clin Pharmacol Ther 66: 246-257

Murthy U, Basu A, Rodeck U, Herlyn M, Ross AH, Das M (1987) Binding of an antagonistic monoclonal antibody to an intact and fragmented EGFreceptor polypeptide. Arch Biochem Biophys 252: 549-560

Ng CM, Bruno R, Combs D, Davies B (2005) Population pharmacokinetics of rituximab (anti-CD20 monoclonal antibody) in rheumatoid arthritis patients during a phase II clinical trial. J Clin Pharmacol 45: 792-801

Pan W (2001) Akaike's information criterion in generalized estimating equations. Biometrics 57: $120-125$

Ritter CA, Arteaga CL (2003) The epidermal growth factor receptortyrosine kinase: a promising therapeutic target in solid tumors. Semin Oncol 30: 3-11

Ross JS, Gray K, Gray GS, Worland PJ, Rolfe M (2003) Anticancer antibodies. Am J Clin Pathol 119: 472-485

Rusch V, Baselga J, Cordon-Cardo C, Orazem J, Zaman M, Hoda S, McIntosh J, Kurie J, Dmitrovsky E (1993) Differential expression of the epidermal growth factor receptor and its ligands in primary non-small cell lung cancers and adjacent benign lung. Cancer Res 53: 2379-2385

Salomon DS, Brandt R, Ciardiello F, Normanno N (1995) Epidermal growth factor-related peptides and their receptors in human malignancies. Crit Rev Oncol Hematol 19: 183-232

Stern M, Herrmann R (2005) Overview of monoclonal antibodies in cancer therapy: present and promise. Crit Rev Oncol Hematol 54: 11-29

Vanhoefer U, Tewes M, Rojo F, Dirsch O, Schleucher N, Rosen O, Tillner J, Kovar A, Braun AH, Trarbach T, Seeber S, Harstrick A, Baselga J (2004) Phase I study of the humanized antiepidermal growth factor receptor monoclonal antibody EMD72000 in patients with advanced solid tumors that express the epidermal growth factor receptor. J Clin Oncol 22: $175-184$

Watanabe T, Shintani A, Nakata M, Shing Y, Folkman J, Igarashi K, Sasada R (1994) Recombinant human betacellulin. Molecular structure, biological activities, and receptor interaction. J Biol Chem 269: 9966-9973

Wells A (1999) EGF receptor. Int J Biochem Cell Biol 31: 637-643

Zhou H, Jang H, Fleischmann RM, Bouman-Thio E, Xu Z, Marini JC Pendley C, Jiao Q, Shankar G, Marciniak SJ, Cohen SB, Rahman MU, Baker D, Mascelli MA, Davis HM, Everitt DE (2007) Pharmacokinetics and safety of golimumab, a fully human anti-TNF-alpha monoclonal antibody, in subjects with rheumatoid arthritis. J Clin Pharmacol 47: $383-396$ 